\title{
Specific leaf area responses to environmental gradients through space and time
}

\author{
John M. Dwyer, ${ }^{1,2,3,4}$ Richard J. Hobbs, ${ }^{2}$ and Margaret M. Mayfield ${ }^{1}$ \\ ${ }^{1}$ University of Queensland, School of Biological Sciences, St. Lucia, Brisbane, Queensland 4072 Australia \\ ${ }^{2}$ School of Plant Biology, University of Western Australia, 35 Stirling Highway, Crawley, Western Australia 6009 Australia \\ ${ }^{3}$ Commonwealth Scientific and Industrial Research Organisation, Ecosystem Sciences, EcoSciences Precinct, Dutton Park, \\ Brisbane, Queensland 4001 Australia
}

\begin{abstract}
Plant communities can respond to environmental changes by altering their species composition and by individuals (within species) adjusting their physiology. These responses can be captured by measuring key functional traits among and within species along important environmental gradients. Some anthropogenic changes (such as fertilizer runoff) are known to induce distinct community responses, but rarely have responses across natural and anthropogenic gradients been compared in the same system. In this study, we used comprehensive specific leaf area (SLA) data from a diverse Australian annual plant system to examine how individual species and whole communities respond to natural and anthropogenic gradients, and to climatically different growing seasons. We also investigated the influence of different leaf-sampling strategies on community-level results. Many species had similar mean SLA values but differed in SLA responses to spatial and temporal environmental variation. At the community scale, we identified distinct SLA responses to natural and anthropogenic gradients. Along anthropogenic gradients, increased mean SLA, coupled with SLA convergence, revealed evidence of competitive exclusion. This was further supported by the dominance of species turnover (vs. intraspecific variation) along these gradients. We also revealed strong temporal changes in SLA distributions in response to increasing growingseason precipitation. These climate-driven changes highlight differences among co-occurring species in their adaptive capacity to exploit abundant water resources during favorable seasons, differences that are likely to be important for species coexistence in this system. In relation to leaf-sampling strategies, we found that using leaves from a climatically different growing season can lead to misleading conclusions at the community scale.
\end{abstract}

Key words: Acacia acuminata; Australia; community assembly; Eucalyptus loxophleba; intraspecific variation; multilevel models; specific leaf area; York gum woodlands.

\section{INTRODUCTION}

Plant communities worldwide are known to vary along a multitude of environmental gradients including shade, soil $\mathrm{pH}$, and soil depth. Increasingly, however, anthropogenic activities impose "new" environmental gradients that include fundamental changes in nutrient supply or disturbance regimes (Vitousek et al. 1997). Plant communities are known to respond in distinct ways to anthropogenic gradients (e.g., fertilization; Hautier et al. 2009), but rarely have responses across natural and anthropogenic gradients been compared in the same system.

Plant community responses to environmental change are mediated to some extent by the functional traits of individual plants in the system (Lavorel and Garnier 2002). In recognition of this, trait-based studies investigate how the distributions of ecologically meaningful functional traits vary among local communities in

Manuscript received 2 March 2013; revised 29 May 2013; accepted 11 July 2013. Corresponding Editor: M. Uriarte.

${ }^{4}$ E-mail: j.dwyer2@uq.edu.au response to environmental gradients or experimental treatments. This approach can yield important information about the dominant community assembly processes operating in a system (Cornwell and Ackerly 2009). More stressful abiotic conditions may reduce the range of species that can persist (Keddy 1992), resulting in trait convergence. Trait convergence may also occur if competitively dominant species with similar trait values exclude competitively inferior species with different trait values (Chesson 2000, Mayfield and Levine 2010). Trait divergence, on the other hand, is best explored after the influences of abiotic factors have been accounted for (Cornwell and Ackerly 2009). Such "residual" divergence indicates niche partitioning, where species with dissimilar trait values (e.g., reflecting different resource acquisition strategies) are more likely to coexist. Shifts in community mean trait values are also informative, and like trait convergence, they can reflect both abiotic filtering and competitive exclusion. Additional insights can be gained by recognizing that trait variation among communities can emerge from two sources: (1) from differences in species composition (interspecific trait 
variation), and (2) from individual specialization within species (intraspecific trait variation). The relative contributions of these two sources may vary depending on the environmental gradient in question, providing further insights about the processes driving community variation (Violle et al. 2012).

Leaf traits have been shown to successfully capture the broad spectrum of leaf investment strategies observed worldwide (Wright et al. 2004), and their strong links with climate and other abiotic conditions make them suitable traits to examine plant community responses to the environment (Lavorel and Garnier 2002). Specific leaf area (SLA) is key among these leaf traits. It is the ratio of the light-capturing surface of a leaf per unit investment of dry mass and is commonly expressed as $\mathrm{mm}^{2} / \mathrm{mg}$. Low-SLA species invest more dry matter per leaf and often have low relative growth rates and net rates of photosynthesis, but have longer leaf life spans (Wright and Westoby 2000, Shipley et al. 2005). High-SLA species adopt a more "disposable" strategy, investing less dry matter per leaf, growing quickly, and having shorter leaf life spans. Evergreen perennial species typically have lower SLA, especially in drier climates and on nutrient deficient soils, where it is important to maintain leaf function when conditions are unfavorable for leaf production (Fonseca et al. 2000, Ordonez et al. 2009). Herbaceous species tend to have high SLA, particularly annual species that have evolved to grow rapidly and reproduce during discrete growing seasons (Garnier 1992). However, even within annual plant ecosystems there can be considerable SLA variation among species, reflecting local-scale spectrums of leaf investment strategies (Huxman et al. 2008). Such SLA variation can become structured at local scales depending on local conditions. For example, in herbaceous plant communities, nutrient enrichment (nitrogen $[\mathrm{N}]$ and phosphorus $[\mathrm{P}]$ ) and disturbance (e.g., grazing) can shift community dominance toward species with higher SLA (e.g., McIntyre 2008, Laliberté et al. 2012).

Specific leaf area also varies intraspecifically and appears to be more influenced by local environmental variation than other leaf traits (e.g., leaf dry matter content; Messier et al. 2010). In herbaceous species, SLA generally increases in response to shade, soil nutrient enrichment (especially $\mathrm{N}$ ), and increased water availability, but these effects can be interactive (Meziane and Shipley 1999, Galmes et al. 2005). Shade-induced increases in SLA are compensatory responses that allow plants to maintain net photosynthetic rates in low-light environments (Evans and Poorter 2001). Increases in SLA associated with water and soil nutrient additions reflect more opportunistic responses that often translate to faster growth, but the magnitude of these responses can be contingent on light levels (Meziane and Shipley 1999). Thus species can differ in their average SLA values (i.e., interspecifically) and also in the manner in which their SLA responds to environmental variation (i.e., their intraspecific SLA responses), and both of these sources of variation are likely to be important for coexistence at the community level (Chesson 2000).

Intraspecific SLA variation has been well documented in some systems (e.g., Kazakou et al. 2007), but this work has focused on variation through space, and not over time. SLA is also known to vary temporally (Angert et al. 2007), but to our knowledge, no studies have explored how community SLA distributions are influenced by temporal intraspecific SLA variation.

In this study we investigate SLA in diverse annual plant communities that occur along a pronounced mean annual precipitation gradient in southwestern Australia. The study system is the winter annual understory component of York gum (Eucalyptus loxophleba)-jam (Acacia acuminata) woodland, a formerly extensive mediterranean ecosystem that now persists only in small, isolated remnants throughout the agricultural region known as the Wheatbelt. Soils of the region are particularly low in plant-available $\mathrm{P}$ (Lambers et al. 2011), but $\mathrm{P}$ and $\mathrm{N}$ enrichment and exotic plant invasions are common where remnants adjoin fertilized crop fields or pastures (Scougall et al. 1993). In addition to these anthropogenic influences, the annual communities also grow along natural local gradients of shade (from completely open to very shaded) created by the patchy Eucalyptus and Acacia canopy. We examine how the SLA of individual species varies along natural and anthropogenic environmental gradients and in response to different growing-season precipitation. We then explore how these species-specific responses transfer to the community scale (Fig. 1). Specifically, we ask the following questions: (1) How do SLA-environment relationships vary among species through space and over time? (2) At the community scale, how do SLA distributions change along natural and anthropogenic gradients? (3) Is the relative contribution of intraspecific variation (vs. species turnover) greater along natural or anthropogenic environmental gradients?

In answering these questions, we also compare community-level results using two alternative leafsampling approaches. First, we calculate species mean SLA values using only sun-exposed leaves. This approach reflects the historical focus on sun leaves for SLA measurements to capture species-level differences (Westoby 1998). Second, recognizing that SLA may vary interannually depending on growing-season precipitation, we calculate species means using "dry year" leaves and apply them to "wet year" communities. We therefore pose a further question: (4) How do different leaf-sampling approaches influence the results of community-scale analyses?

\section{Methods \\ Field surveys}

Community surveys were undertaken in the understory of York gum woodlands throughout the wheatbelt in southwestern Australia during the 2010 and 2011 growing seasons. The study region extended approxi- 


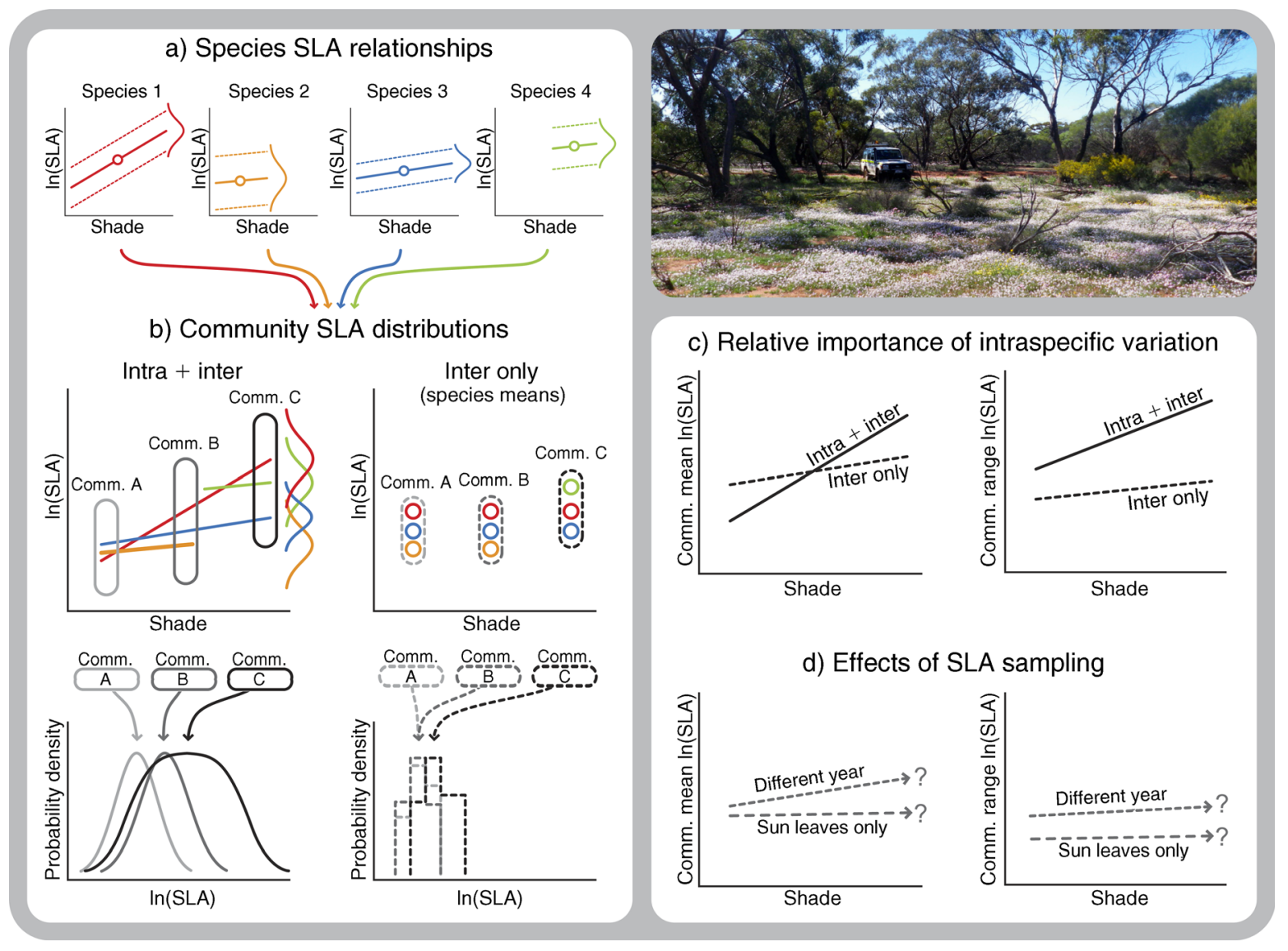

FIG. 1. Four aspects of specific leaf area $\left(\mathrm{SLA} ; \mathrm{mm}^{2} / \mathrm{mg}\right)$ variation explored in this community survey undertaken in the understory of York gum woodlands in southwestern Australia during the 2010 and 2011 growing seasons, and a diagrammatic representation of the analyses used. Hypothetical relationships with percentage shade are shown as an example. In panel (a) solid lines are fitted relationships, dotted lines are $95 \%$ confidence intervals, and curves indicate the amount of variance not explained by the measured environment. Open circles represent mean SLA values for each species. These symbols are also used in panel (b) to show how species-level relationships translate to the community (Comm.) scale. The density plots in the lower part of panel (b) indicate how community SLA distributions were generated for the "intra + inter" (intraspecific and interspecific trait variation) and "inter only" approaches. These distributions were then characterized by their mean and range, which in turn were modeled in relation to environmental variables [panel (c)]. Panel (d) illustrates how different leaf-sampling strategies may influence communitylevel analyses. The photograph shows a York gum woodland in bloom. Photo by John M. Dwyer.

mately from Quairading in the southwest to Perenjori in the northeast. Detailed methods are provided in Appendix A. In brief, communities were sampled in $0.09-\mathrm{m}^{2}$ quadrats in a spatially nested design; fifteen quadrats were randomly located within $225-\mathrm{m}^{2}$ sites within woodland remnants. Remnants were public reserves or fenced woodland patches retained on private properties. Only remnants in the north of the study region (comprising 30 sites and 450 quadrats) were sampled in both years. In 2010, herbaceous cover was insufficient to undertake sampling in the south of the region due to well-below average rainfall. In the wetter 2011 season, an additional nine remnants (comprising 47 sites and 705 quadrats) were sampled in the south. In each quadrat the identity and abundance of all species were recorded, and the tallest specimen of each species was collected and pressed in the field. Soil samples were also collected from each quadrat and later analyzed for ammonium, nitrate, plant-available $\mathrm{P}$, and $\mathrm{pH}$. Ammonium and nitrate were combined into one variable approximately representing plant-available N. Woody canopy cover and the presence of residual dry grass matter (RDGM; from exotic annual grasses) were recorded for each quadrat. Of these measured environmental variables, $\mathrm{P}$ and RDGM capture human-created conditions associated with nearby agricultural land uses.

\section{SLA measurements}

We took specific leaf area (SLA) measurements on field-collected specimens back in the laboratory. One fully expanded healthy leaf, including the petiole, was selected from the top half of each specimen, regardless of how sun exposed the specimen was (as indicated by woody-cover values for each quadrat, which ranged from $0 \%$ to $99 \%$ in this open woodland system; Fig. 1). In some cases no healthy leaves were available, in which 
case the specimen was not sampled. Selected leaves were rehydrated following Cornelissen et al. (2003) prior to digital area measurement. Each leaf was then oven-dried at $60^{\circ} \mathrm{C}$ for $72 \mathrm{~h}$ and weighed using a microbalance (Sartorius AG, Goettingen, Germany). A total of 4850 SLA measurements were made on 190 species over two growing seasons; this included $>100$ measurements each for common species.

\section{Statistical analyses}

Our analytical approach is conceptualized in Fig. 1 and described in this section.

Species-specific models.-Sufficient SLA measurements were available to test intraspecific SLA-environment relationships for 85 species that comprised $93 \%$ of all individuals recorded over the two survey years (Appendix B and C). Relationships were quantified separately for each species using multilevel linear models that appropriately captured the spatially nested survey design and also accounted for the variable numbers of SLA values per site (within-site $n$ ranged from 1 to 15 ; Gelman and Hill 2007). SLA was ln-transformed prior to all analyses to meet the assumptions of linear modeling and two explanatory variables were also transformed to improve linearity of relationships. Explanatory variables corresponded mostly to the quadrat scale and were selected to represent the localscale growing environment. These include woody cover, $\ln (\mathrm{N})$, square-root-transformed $\mathrm{P}$, and $\mathrm{pH}$. We also included growing-season precipitation at the remnant scale to capture regional climate effects. For species with at least eight measurements in each year, we also included a binary indicator variable for year. A series of candidate models with different combinations of variables was fit for each species using maximum likelihood estimation. Because precipitation was $\sim 100$ $\mathrm{mm}$ greater at all sites in 2011 (i.e., year and precipitation were correlated), we did not consider models with both year and precipitation included. Instead, these variables were included in separate "sets" of models (each variable in combinations with quadratscale variables), and both of these sets were included in the candidate models for each species. Candidate models were compared using $\mathrm{AIC}_{\mathrm{c}}$ values following Burnham and Anderson (2002). In all cases remnant and site (within remnant) were included as random effects. The model with most support for a given species was refit using restricted maximum likelihood (REML), and coefficient estimates were recorded. The within-site (residual) variance was also recorded and used in subsequent analyses to represent "local-scale variation," i.e., local-scale variation among quadrats and within plants. Because only one leaf was measured on any given plant, it was not possible to partition this variation into separate among- and within-plant components.

Generating community trait distributions.-Leaves from many collected specimens could not be sampled due to herbivore or mold damage. To overcome these data gaps, we used the species-specific models to predict $\ln$ (SLA) values for every species occurrence, based on values of the environmental variables associated with each occurrence. Instead of using the overall (fixed effect) intercept for these calculations, we used weighted site intercepts (best linear unbiased predictors at the site level) to account for unexplained among-site differences. These predicted values were used only for the 85 species with enough measurements to be modeled intraspecifically. For remaining species, we used year means or overall means (if measured in one year only) and applied these mean values to all occurrences. The $R^{2}$ value for measured vs. predicted values was 0.67 (intercept 0.0, slope 1.0).

Community $\ln$ (SLA) distributions were generated for each quadrat (community) using four distinct approaches. For the first approach, which we refer to as "intra + inter," we used the predicted $\ln$ (SLA) values for each species in each quadrat. We also incorporated local-scale intraspecific variation in each quadrat's distribution. To do this we simulated $5000 \ln$ (SLA) distributions for each quadrat. In each simulation, $\ln$ (SLA) values for each species were drawn from their own normal distribution, with mean equal to the predicted value and variance equal to the within-site variance (from the species models; Appendix B). The number of draws from each species' distribution corresponded to the observed species abundances in each quadrat. For each simulated distribution we calculated the mean and range. We then used the medians of these metrics from the 5000 simulations as our estimated community mean and community range values for each quadrat. Ranges were calculated to provide an indication of SLA convergence or divergence along environmental gradients.

For the remaining approaches, we used each species' mean $\ln$ (SLA) value (calculated from actual measurements) and applied it to every occurrence of a species regardless of environmental conditions. Species means were calculated in three ways: (1) as the mean $\ln$ (SLA) value of a species calculated separately for each survey year; (2) as the mean for each year, but only using sunexposed leaves; and (3) as the mean for the drier sampling season (2010) only. We refer to these approaches as "inter only," "inter only (sun)," and "inter only (dry)" respectively. We defined "sunexposed" thresholds separately for each species as the lower 25th percentile of woody-cover values for the quadrats in which they occurred. Because some species had zero (or very few) measurements for a given scenario, we applied whatever mean value was available for the species (mostly the 2011 mean). Refer to Appendix $\mathrm{B}$ and $\mathrm{C}$ for more information. In all approaches we included all species for which SLA measurements were available (190 species).

Models of community means and ranges.-The final step in our analysis was to assess relationships between the environment and the community means and ranges. Once again, we used multilevel linear models estimated 
using REML. In all models, the following predictors were included as additive terms at the quadrat level: woody cover, square-root-transformed $\mathrm{P}, \ln (\mathrm{N}), \mathrm{pH}$, and RDGM. Growing-season precipitation was included at the remnant level. To permit comparison of estimated parameters, all explanatory variables were standardized by mean-centering and dividing by two standard deviations (Gelman 2008). We generated 95\% highest posterior density (HPD) intervals using Markov chain Monte Carlo sampling methods (Bates and Maechler 2009). In addition, we tested for differences in community means and ranges between the 2010 and 2011 growing seasons using only the remnants that were sampled in both years. For this analysis we ran multilevel ANOVAs with year included as the sole fixed effect. Year was also included in the random-effects structure along with remnant, site, and quadrat to account for repeated sampling of quadrats over the two years. Statistical analyses were done in R ( R Development Core Team 2012). All multilevel linear models were fit using the lme4 package (Bates and Maechler 2009), and all model comparisons were undertaken using the model selection function in the MuMIn package (Bartoń 2012).

\section{RESUlts}

Species SLA relationships.-Of the 85 species for which specific leaf area (SLA)-environment relationships could be assessed, 61 had significant associations with at least one environmental variable (Appendix B). Fifty-one species had measurements that sufficiently spanned the two growing seasons, and around half of these had significantly higher SLA values in the wetter 2011 growing season. For a further 19 species, growingseason precipitation (within and across growing seasons) was a better predictor than the year indicator variable, and these precipitation relationships were all positive. Woody cover was the most common significant localscale explanatory variable. It was included in selected models for 25 species and had a positive association with SLA in all cases (Appendix B). Fig. 2 shows selected significant relationships based on species-specific models. Of note is the large amount of unexplained SLA variation (also refer to $R^{2}$ values in Appendix B).

Community SLA distributions.-Using the "intra + inter" approach applied to 2011 data (the more spatially extensive data set), all measured environmental variables had positive and significant relationships with community mean $\ln (\mathrm{SLA})$ except for growing-season precipitation that was not significant (Figs. $3 a$ and $4 a-c)$. Environmental variables had varied effects on the range of community $\ln$ (SLA) distributions. Woody cover and $\ln (\mathrm{N})$ had no discernable effect, $\mathrm{pH}$ and growing-season precipitation were positively related, and square-roottransformed $\mathrm{P}$ and $\mathrm{RDGM}$ were negatively related (Figs. 3b and 4d-f). Importantly, $P$ enrichment and the presence of annual grass litter (the anthropogenic factors) were the only variables associated with positive mean shifts and SLA convergence.

On average, each remnant received an additional 100 $\mathrm{mm}$ of precipitation in 2011 compared with 2010. This substantial increase in precipitation was associated with strong increases in both the mean and range of community $\ln$ (SLA) distributions (Fig. 5).

Relative importance of intraspecific variation.-The contribution of intraspecific variation along environmental gradients is evident in the difference in slope estimates between the "intra + inter" and "inter only" approaches (Figs. 1 and 3; Cornwell and Ackerly 2009). The slope estimate for woody cover was significantly higher using the "intra + inter" approach (no overlap in the highest posterior density [HPD] intervals of slope estimates). This indicates that intraspecific variation contributed substantially to overall community responses to shade, which is not surprising given that woody cover had a positive effect on SLA in many speciesspecific models (Appendix B). Slope estimates for $\ln (\mathrm{N})$ and $\mathrm{pH}$ were also higher using the "intra + inter" approach, but the difference in slope estimates between the two approaches was not as pronounced. Slope estimates for square-root-transformed $\mathrm{P}$ and RDGM were similar using both approaches, showing that species turnover is mainly driving increases in community means along these human-created gradients. Model intercepts did not differ significantly between the "intra + inter" approach (estimate $=3.5, \mathrm{SE}=0.008)$ and "inter only" approach (estimate $=3.51, \mathrm{SE}=0.008$ ).

Differences in slope estimates between the "intra + inter" and "inter only" approaches were less pronounced in the models of community ranges. The largest difference was seen for growing-season precipitation, which indicated strong intraspecific contributions to SLA range increases along spatial precipitation gradients (Fig. 3b). The intercept for the "intra + inter" approach was much higher than for the "inter only" approach (Fig. 4d-f). This higher intercept, reflecting higher overall range values, was not due to intraspecific responses to environmental gradients, but instead due to the inclusion of local-scale (within-site and within-plant) variation that was independent of the measured environment.

The temporal changes in community SLA distributions could have originated from three sources: changes in species' abundances (absolute and relative), intraspecific responses to growing-season rainfall, and the appearance of new species in the wetter 2011 season. The contribution of new species is a possibility because 2011 communities had an average of 4.1 more species per quadrat (95\% HPD intervals, 3.2-4.9), due in part to the emergence of species that were not recorded in 2010. To decompose these sources we used a two-step approach. First, we generated community SLA distributions for the 2011 communities using only species that were recorded in both years (i.e., we removed the influence of new species). Second, we generated 2010 

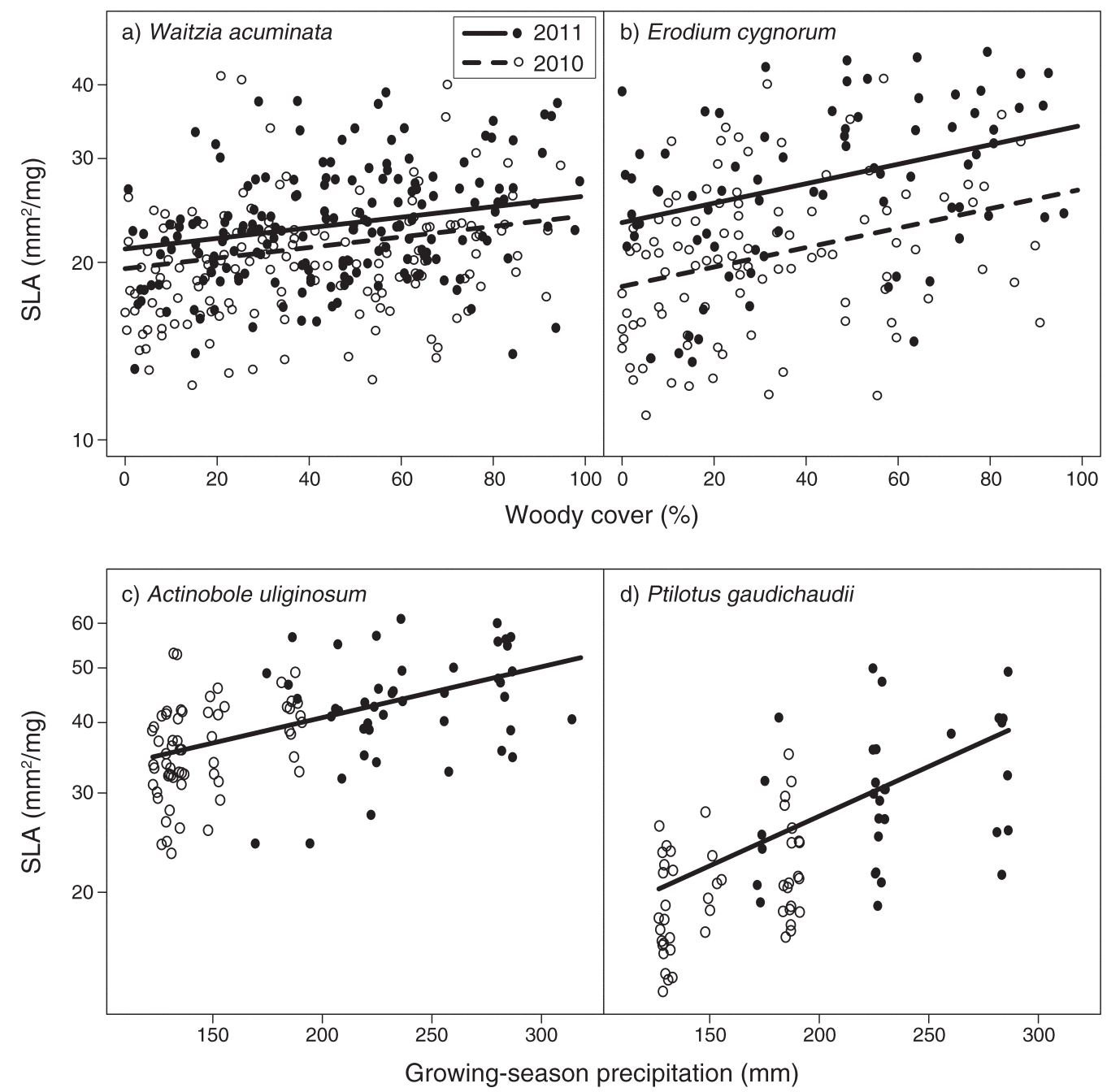

FIG. 2. Selected plots from species-specific models of $\ln ($ SLA): woody cover vs. SLA of (a) Waitzia acuminata (Asteraceae) and (b) Erodium cygnorum (Geraniaceae), with separate lines for each year; and growing-season precipitation (across years) vs. SLA of (c) Actinobole uliginosum (Asteraceae) and (d) Ptilotus gaudichaudii (Amaranthaceae). Fitted lines are from the restricted maximum-likelihood estimated multilevel models. Open circles indicate 2010 values, and solid circles indicate 2011 values. Note the $y$-axis $\log$ scale.

and 2011 communities using only species means from 2010 (the "inter only (dry)" approach), and compared these to communities generated from the "intra + inter" approach to gauge the contribution of both abundance changes and intraspecific responses. Using the first step we found almost no difference in the mean and range compared to the complete 2011 community distributions (Fig. 5a, c), indicating that new species contributed little to the observed distributional changes. The second step revealed that both abundance changes and intraspecific responses contributed substantially to the changes (Fig. $5 a-d)$. Predictably, intraspecific variation contributed most $(70 \%)$ to temporal changes in community SLA ranges. The implication of lower community means and ranges in 2010 is that relative SLA differences among cooccurring individuals are substantially smaller during dry growing seasons compared to wet growing seasons.
Effects of leaf sampling.-We used the "inter only (sun)" and "inter only (dry)" approaches applied to 2011 communities to respectively assess the effects of using only sun-exposed leaves or leaves from a drier growing season. Because these approaches do not incorporate intraspecific variation, we compared them only to the "inter only" approach. For models of community mean $\ln$ (SLA), slope estimates using "inter only (sun)" were generally similar to those using "inter only"; however, slopes using "inter only (dry)" were considerably lower, to the extent that most were not significant (Fig. 3a). The "inter only (dry)" approach also reduced the intercept substantially (Fig. 4d-f), which was expected given the lower SLA values observed in many species in 2010. Differences among approaches were far less pronounced in models of community $\ln$ (SLA) ranges. In summary, using SLA values from a climatically 

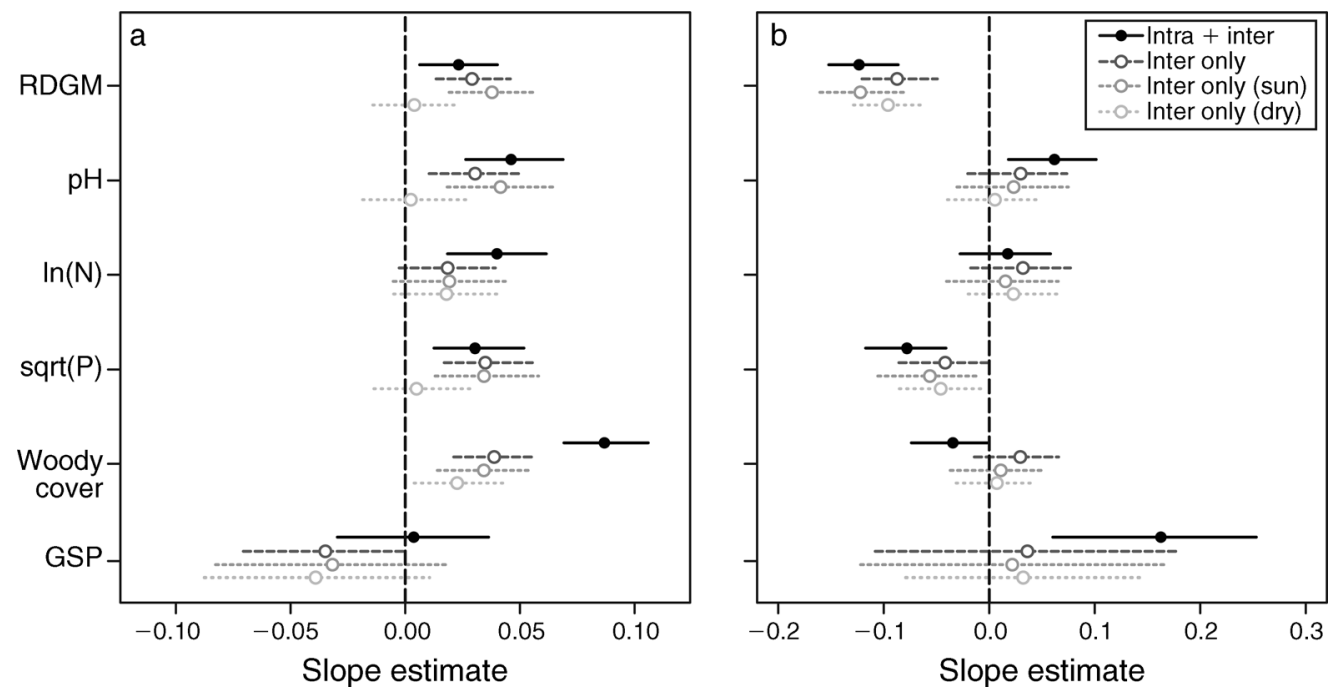

FIG. 3. Estimated slope coefficients from models of (a) community mean $\ln$ (SLA) and (b) community range of $\ln$ (SLA). Symbols indicate intraspecific and interspecific trait variation, and the terms "inter only (sun)" and "inter only (dry)" indicate the scenarios where species mean SLA values were calculated using only sun-exposed leaves and only leaves from the drier sampling year, respectively. Horizontal bars indicate $95 \%$ highest posterior density (HPD) intervals for each slope estimate. Variables include: RDGM, residual dry grass matter; $\ln (\mathrm{N})$, ln-transformed nitrogen; sqrt(P), square-root-transformed phosphorus; and GSP, growing-season precipitation.

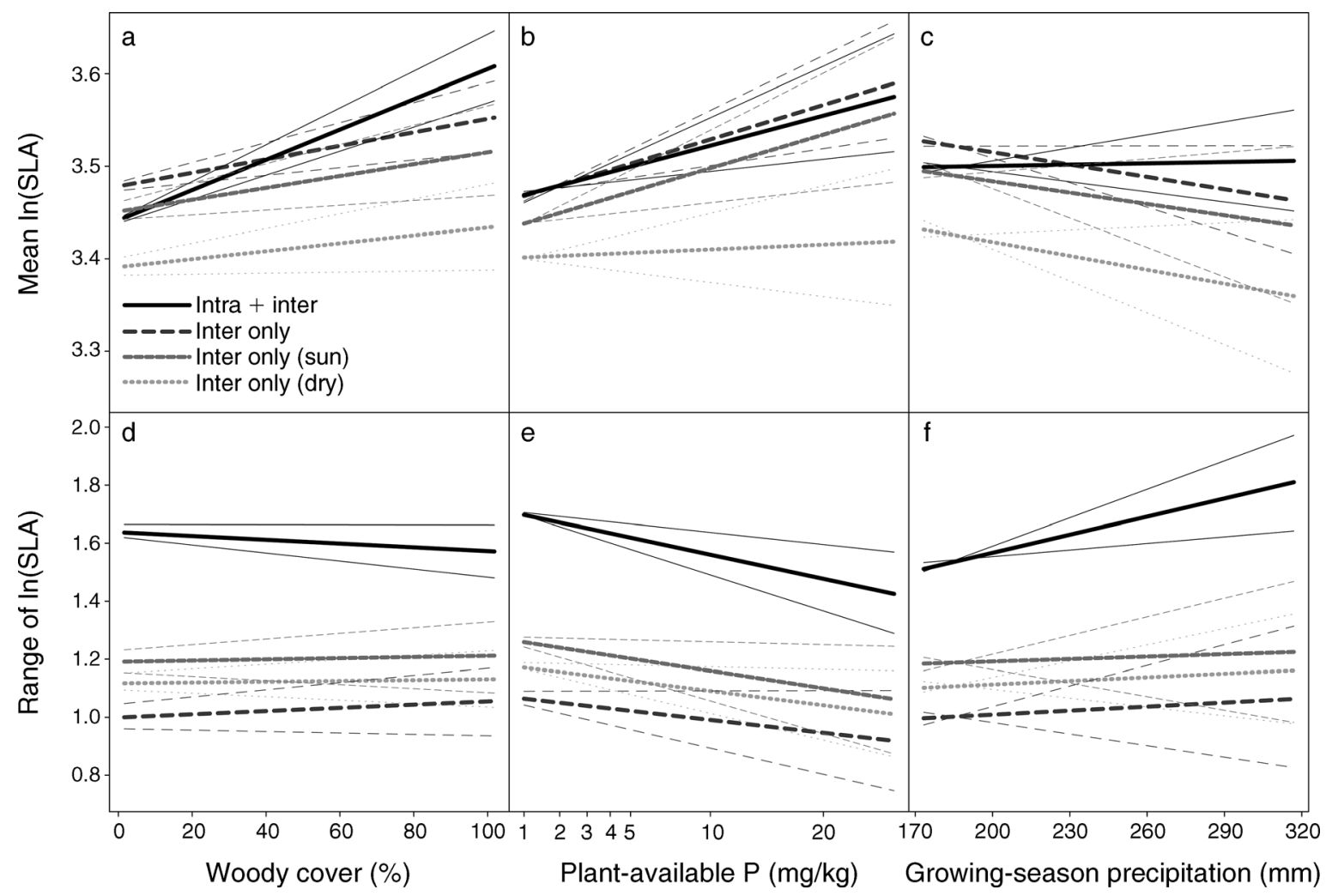

FIG. 4. Selected plots from multilevel linear models of (a-c) community mean $\ln (\mathrm{SLA})$ and (d-f) community range of $\ln$ (SLA). Separate lines were fit for each SLA $\left(\mathrm{mm}^{2} / \mathrm{mg}\right)$ measurement scenario. Thick lines were fit using point estimates, and thin lines are 95\% CI, reflecting uncertainty in both the slope and intercept of each relationship. The terms "inter only (sun)" and "inter only (dry)" indicate the scenarios where species mean SLA values were calculated using only sun-exposed leaves and only leaves from the drier sampling year, respectively. 
2010-2011 change in community means:

Overall change (on log scale) $=$ (2) - (4)

Average contributions of each source of change:
(2) to (3) New species in $2011=0 \%$
(4) to (5) Abundance changes $=54 \%$
(5) to (3) Intraspecific variation $=46 \%$

a) Community means: intra + inter

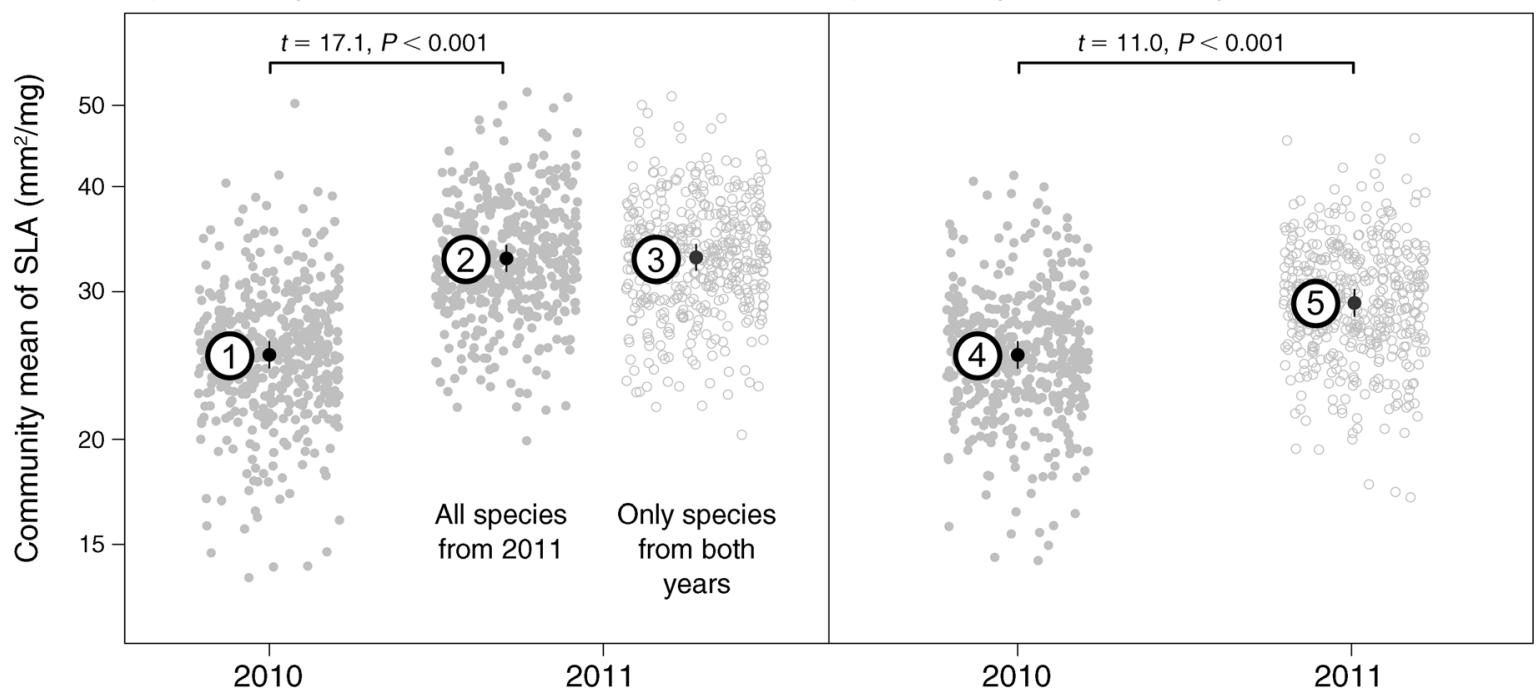

2010-2011 change in community ranges:

Overall change $=$ (7) - (9)

Average contributions of each source of change:

(7) to (8) New species in $2011=4 \%$

(9) to (10) Abundance changes $=26 \%$

(10) to (8) Intraspecific variation $=70 \%$

c) Community ranges: intra + inter

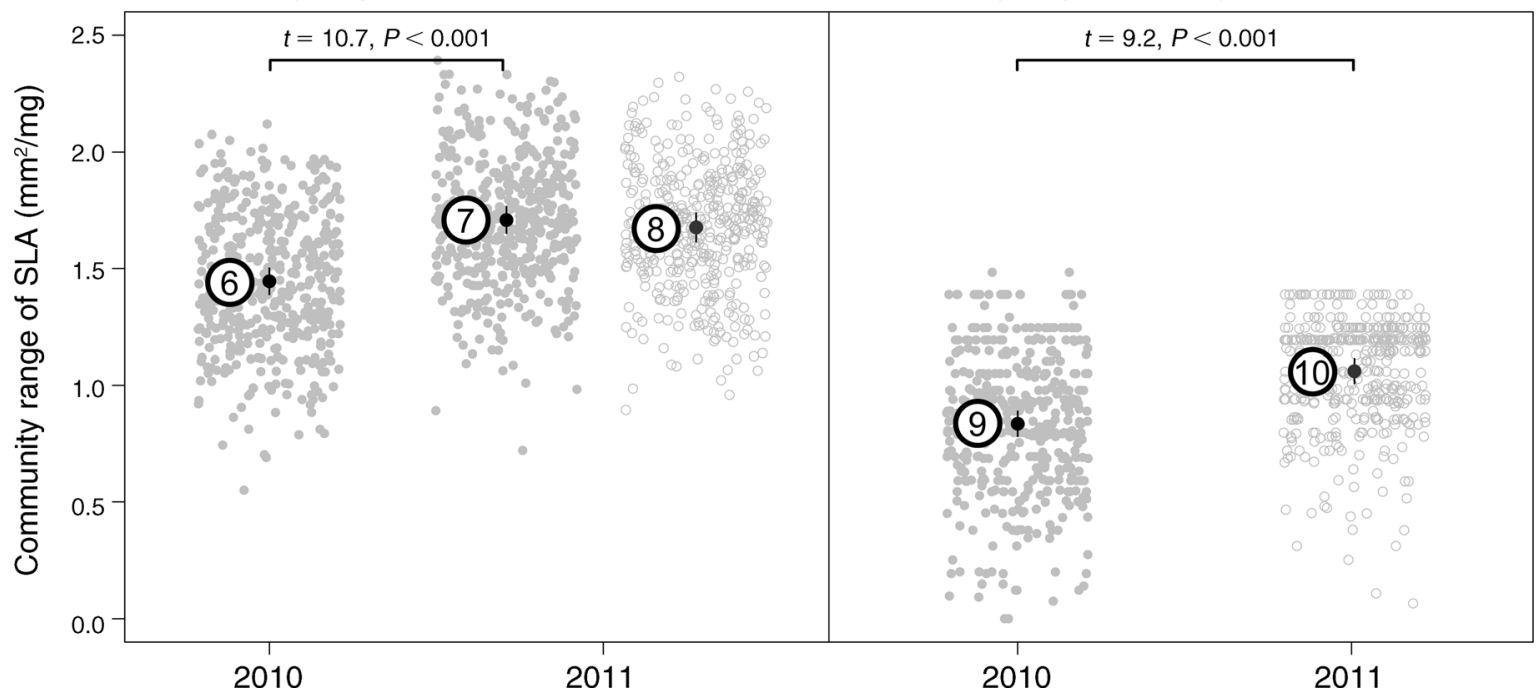

FIG. 5. Between-year comparisons of ( $a, b)$ community means and (c, d) ranges, showing the decomposition of the temporal SLA response into three sources: new species in 2011 , abundance changes, and intraspecific variation. In all panels, light gray points are individual community (quadrat) values, black points are means for each year (calculated from multilevel ANOVAs), and bars are corresponding 95\% HPD intervals. Test statistics ( $t$ values and associated $P$ values) for year effects are included for most comparisons. Plots (a) and (c) show community means and ranges derived from the "intra + inter" approach. The open points 
different growing season had a larger effect on community-level results than using sun-exposed leaves.

\section{Discussion}

This study demonstrates that co-occurring species can have similar mean specific leaf area (SLA) values, but differ in their SLA responses to environmental variation through space and time. At the community scale, we found that intraspecific variation contributes to substantial changes in community SLA distributions in response to growing-season precipitation, such that relative differences among co-occurring individuals are smaller during drier growing seasons. We also found that communities respond differently to natural and anthropogenic gradients. Along anthropogenic gradients ( $P$ and residual dry grass matter [RDGM]), increased mean SLA coupled with SLA convergence revealed evidence of competitive exclusion. This was further supported by the dominance of species turnover (vs. intraspecific variation) along these gradients. In relation to leaf-sampling strategies, we found that using leaves from a climatically different growing season can have misleading effects on community-level SLA results.

How do SLA-environment relationships vary among species through space and over time? - Based on previous experimental work on herbaceous plant species (Knops and Reinhart 2000, Galmes et al. 2005), we anticipated that many of our study species would have positive SLA relationships with precipitation, shade, and soil $\mathrm{N}$. Consistent with this expectation, half of the modeled species had significantly higher SLA in the wetter growing season or had significant positive relationships with precipitation within and across years, and $30 \%$ of species showed positive relationships with shade. However, only $13 \%$ of modeled species showed significant SLA relationships with soil $\mathrm{N}$. There are a number of reasons why relationships with $\mathrm{N}$ may not have been as apparent as expected. First, shade and soil nutrients can have interactive effects on SLA such that nutrient effects are mainly evident in shaded situations (Meziane and Shipley 1999). Due to small sample sizes for some species, we did not include interactions in our candidate models, so some of the SLA variation attributed to shade may be due to soil N. Second, we may have underestimated $\mathrm{N}$ because soil was sampled late in the growing season, by which time $\mathrm{N}$ (especially nitrate) may have been leached by rainfall events or depleted by plant growth (Prober et al. 2005).

Regarding soil $\mathrm{P}$, we anticipated that native species adapted to low-P soils would not show SLA responses to $\mathrm{P}$ enrichment, and this was indeed what we found. Only four native species had significant SLA relationships with soil P (Appendix B), all mildly positive. This was despite the fact that many well-sampled native species occurred across a range of $\mathrm{P}$ conditions from low to high (e.g., Erodium cygnorum, Goodenia berardiana, Waitzia acuminata; Appendix D). Phosphorus has been shown to increase SLA in herbaceous plant species, but only when $\mathrm{N}$ is in abundant supply (Sims et al. 2012), and this might explain the general insensitivity to $\mathrm{P}$ that we observed. Interestingly, none of the "exploitative" exotic species in our system, such as Avena barbata or Brassica tournefortii, had significant SLA relationships with $\mathrm{P}$ or N.

Unexplained variance was considerable for many species (Fig. 2; Appendix B) and is probably due to unmeasured environmental variables (e.g., finer scale shading effects from neighboring plants), leaf age effects, and "random" phenotypic variation.

At the community scale, how do SLA distributions change along natural and anthropogenic gradients?-The generally positive responses of individual species to shade and precipitation were expected to translate to the community scale as distributional shifts to higher mean SLA values in shadier, wetter locations. We found this for shade (Figs. 3 and 4), but positive precipitation responses were apparent only between years, not along spatial precipitation gradients in a given year, presumably because conditions were uniformly bad in 2010 and uniformly good in 2011. The dramatic increases in community means and ranges in 2011, which emerged largely from intraspecific responses (Fig. 5), are particularly interesting. The increasing means logically indicate widespread increases in water exploitation (via leaf area expansion), and hence increased relative growth rates, which is corroborated by community biomass and height data (J. M. Dwyer, R. J. Hobbs, and M. M. Mayfield, unpublished data). The increasing community ranges point to differences among cooccurring (i.e., potentially interacting) species in their adaptive capacity to exploit abundant water resources, and these differences are likely to be very important for species coexistence. In the Sonoran Desert, for example, winter annuals display a similar spectrum of abilities to exploit soil water via SLA adjustments (Angert et al. 2007). Importantly, a tradeoff exists between exploitative ability and water-use efficiency (Angert et al. 2009, Angert et al. 2010), and this trade-off provides opportunities for species to differ in their demographic responses to growing-season precipitation, thereby promoting species coexistence via the storage effect (Chesson 2000, Angert et al. 2009). While we have not

in panels (a) and (c) show 2011 communities generated using only the species that were present in both years. Plots (b) and (d) show community means and ranges generated using species means from 2010 applied to both years. In these plots, only species from both years are included in the 2011 communities, as indicated by the open points for 2011. Beside the plots are calculations of the contributions of each source of temporal change. Numbers beside each estimated mean in panels (a-d) are included to illustrate how the various contributions were calculated for community means (upper) and community ranges (lower). 
explicitly demonstrated such a tradeoff in our study system, we have shown that species differ in their SLA responses to growing-season precipitation, and that these differences manifest themselves clearly in community SLA distributions. To investigate a possible link between exploitative ability and demography in our data, we assessed the relationship between the magnitude of SLA increases (from 2010 to 2011) and changes in species' relative and absolute abundances over the same period. Regardless of the measure of abundance used, we found no evidence of such a link (Appendix E: Fig. E1), perhaps not surprising given that our data span only two growing seasons.

Consistent with previous studies in P-limited herbaceous communities (Laliberté et al. 2012), we observed community shifts to high SLA species in response to P enrichment. In the present study, shifting mean SLA values were coupled with SLA convergence, providing strong evidence for competitive exclusion by exploitative, high-SLA species. More specifically, it reflects intensifying light competition following release from nutrient limitation (Hautier et al. 2009) at the expense of lower SLA species. Also consistent with previous work, the distributional changes in response to $\mathrm{P}$ were driven by changes in composition, in our case from nativedominated to exotic-dominated communities (J. M. Dwyer, R. J. Hobbs, and M. M. Mayfield, unpublished data).

Is the relative contribution of intraspecific variation (vs. species turnover) greater along natural or anthropogenic environmental gradients? - The contribution of intraspecific variation, relative to species turnover, was greatest along local woody-cover gradients (Fig. 3), and was also very pronounced in response to different growing-season precipitation (Fig. 5). This is not surprising given the significant positive relationships with shade and precipitation (or year) found for many common and abundant species (Appendix B). We cannot say how much of the observed intraspecific variation was due to phenotypic plasticity vs. genetic differences, but given the local scale of the woody-cover gradient and the well-demonstrated plasticity of herbs in response to shade and water availability (Sultan and Bazzaz 1993), it is likely that plasticity is important in this system. By contrast, community SLA responses to the anthropogenic factors were driven mainly by species turnover. These different community responses indicate that native species are able to respond intraspecifically to gradients along which they have evolved, like shade, precipitation, and $\mathrm{N}$, but not to "new" gradients associated with recent land-use change ( $\mathrm{P}$ enrichment and RDGM). At the same time, many of the introduced species in the system are highly competitive (e.g., Avena barbata; Liancourt et al. 2009) and are preadapted to exploit high nutrient situations (e.g., Bromus rubens; Brooks 2003), thereby driving species turnover along the anthropogenic fertility gradient. Most of the exotic annual grasses also produce litter (RDGM), which facilitates their persis- tence and reduces the germination and establishment of co-occurring species (Lenz et al. 2003). Some of these species also germinate high proportions of their seed each growing season (Stevens et al. 2007) and probably outcompete native species that do manage to germinate in the litter (Standish et al. 2008). This combination of abiotic changes and the introduction of preadapted, competitive species is, of course, not unique to our system (Hobbs et al. 2009), but our findings provide new insights into the processes driving community responses to this common land-use change scenario.

How do different leaf-sampling approaches influence the results of community-scale analyses?-We anticipated that using only sun-exposed leaves would underestimate community mean SLA responses because many of the species-specific models identified lower SLA values in open situations. However, we found that this approach only marginally reduced community mean estimations (evident in the slightly lower intercepts in Fig. $4 a-c$ ). In addition, the estimated relationships with environmental variables were very similar to those from the "inter only" approach (Fig. 3a). This indicates that in this herbaceous system, relative species differences are captured whether or not shade leaves are excluded from species mean SLA calculations. The use of leaves from a climatically drier growing season had a more pronounced effect at the community scale. This approach dramatically shifted community SLA distributions to lower mean values (much lower intercepts in Fig. 4a-c) and tended to dampen community mean responses along environmental gradients to the point that almost all explanatory variables would have been deemed unimportant. This dampening occurred because SLA differences among co-occurring species were smaller in the dry year. Our system is unlikely to be unique in this regard, so we therefore warn against using species mean SLA values calculated from climatically different years if the goal is to examine community functional responses along environmental gradients.

\section{Conclusions}

This study highlights the utility of functional traits for investigating the processes driving community responses to environmental change. Obviously, the choice of trait(s) needs to be carefully considered and will depend on the system and the nature of environmental gradients. In this case, a single leaf trait captured contrasting responses to natural and anthropogenic gradients. Importantly, this trait varied among and within species, but in different ways depending on the gradient. It also varied temporally in many of the studied species, resulting in strong community-level shifts across years. We echo recent calls for the inclusion of intraspecific variation in trait-based studies, but extend the challenge also to incorporate temporal trait variation, particularly in systems that experience pronounced climatic variation. 


\section{ACKNOWLedgments}

Thanks to Justine Gay-des-Combes, Hao Ran Lai, Caroline Oldstone-Moore, Emily Searle, and Monica Radovski for assistance with specific leaf area measurements, and to Suzanne Schmidt's lab for access to the microbalance. Thanks also to the World Wildlife Fund for organizing access to Woodland Watch properties, to the landholders for their cooperation, and to government agencies for permitting access to public reserves. We thank Suzanne Prober for sharing her study sites and Mike Hislop and Jenny Borger for assistance with plant identification. We are grateful to Yvonne Buckley and two anonymous reviewers for valuable comments on the manuscript. This research was funded by an Australian Research Council grant (DP1094413) awarded to M. M. Mayfield and R. J. Hobbs.

\section{Literature Cited}

Angert, A. L., J. L. Horst, T. E. Huxman, and D. L. Venable. 2010. Phenotypic plasticity and precipitation response in Sonoran Desert winter annuals. American Journal of Botany 97:405-411.

Angert, A. L., T. E. Huxman, G. A. Barron-Gafford, K. L. Gerst, and D. L. Venable. 2007. Linking growth strategies to long-term population dynamics in a guild of desert annuals. Journal of Ecology 95:321-331.

Angert, A. L., T. E. Huxman, P. Chesson, and D. L. Venable. 2009. Functional tradeoffs determine species coexistence via the storage effect. Proceedings of the National Academy of Sciences USA 106:11641-11645.

Bartoń, K. 2012. MuMIn: Multi-model inference. R package version 1.7.2. http://CRAN.R-project.org/package=MuMIn

Bates, D., M. Maechler, and B. Bolker. 2011. lme4: Linear mixed-effects models using $\mathrm{S} 4$ classes. $\mathrm{R}$ package version 0.999375-42. http://CRAN.Rproject.org/package=lme4

Brooks, M. L. 2003. Effects of increased soil nitrogen on the dominance of alien annual plants in the Mojave Desert. Journal of Applied Ecology 40:344-353.

Burnham, K. P., and D. R. Anderson. 2002. Model selection and multimodel inference: a practical information-theoretic approach. Second edition. Springer-Verlag, New York, New York, USA.

Chesson, P. 2000. Mechanisms of maintenance of species diversity. Annual Review of Ecology and Systematics 31: 343-366.

Cornelissen, J. H. C., S. Lavorel, E. Garnier, S. Diaz, N. Buchmann, D. E. Gurvich, P. B. Reich, H. ter Steege, H. D. Morgan, M. G. A. van der Heijden, J. G. Pausas, and H. Poorter. 2003. A handbook of protocols for standardised and easy measurement of plant functional traits worldwide. Australian Journal of Botany 51:335-380.

Cornwell, W. K., and D. D. Ackerly. 2009. Community assembly and shifts in plant trait distributions across an environmental gradient in coastal California. Ecological Monographs 79:109-126.

Evans, J. R., and H. Poorter. 2001. Photosynthetic acclimation of plants to growth irradiance: the relative importance of specific leaf area and nitrogen partitioning in maximizing carbon gain. Plant Cell and Environment 24:755-767.

Fonseca, C. R., J. M. Overton, B. Collins, and M. Westoby. 2000. Shifts in trait-combinations along rainfall and phosphorus gradients. Journal of Ecology 88:964-977.

Galmes, J., J. Cifre, H. Medrano, and J. Flexas. 2005. Modulation of relative growth rate and its components by water stress in Mediterranean species with different growth forms. Oecologia 145:21-31.

Garnier, E. 1992. Growth analysis of congeneric annual and perennial grass species. Journal of Ecology 80:665-675.

Gelman, A. 2008. Scaling regression inputs by dividing by two standard deviations. Statistics in Medicine 27:2865-2873.
Gelman, A., and J. Hill. 2007. Data analysis using regression and multi-level/hierarchical models. Cambridge University Press, New York, New York, USA.

Hautier, Y., P. A. Niklaus, and A. Hector. 2009. Competition for light causes plant biodiversity loss after eutrophication. Science 324:636-638.

Hobbs, R. J., E. Higgs, and J. A. Harris. 2009. Novel ecosystems: implications for conservation and restoration. Trends in Ecology and Evolution 24:599-605.

Huxman, T. E., G. Barron-Gafford, K. L. Gerst, A. L. Angert, A. P. Tyler, and D. L. Venable. 2008. Photosynthetic resource-use efficiency and demographic variability in desert winter annual plants. Ecology 89:1554-1563.

Kazakou, E., E. Garnier, M. L. Navas, C. Roumet, C. Collin, and G. Laurent. 2007. Components of nutrient residence time and the leaf economics spectrum in species from mediterranean old-fields differing in successional status. Functional Ecology 21:235-245.

Keddy, P. A. 1992. Assembly and response rules - two goals for predictive community ecology. Journal of Vegetation Science 3:157-164

Knops, J. M. H., and K. Reinhart. 2000. Specific leaf area along a nitrogen fertilization gradient. American Midland Naturalist 144:265-272.

Laliberté, E., B. Shipley, D. A. Norton, and D. Scott. 2012. Which plant traits determine abundance under long-term shifts in soil resource availability and grazing intensity? Journal of Ecology 100:662-677.

Lambers, H., M. C. Brundrett, J. A. Raven, and S. D. Hopper. 2011. Plant mineral nutrition in ancient landscapes: high plant species diversity on infertile soils is linked to functional diversity for nutritional strategies. Plant and Soil 348:7-27.

Lavorel, S., and E. Garnier. 2002. Predicting changes in community composition and ecosystem functioning from plant traits: revisiting the Holy Grail. Functional Ecology 16: $545-556$.

Lenz, T. I., J. L. Moyle-Croft, and J. M. Facelli. 2003. Direct and indirect effects of exotic annual grasses on species composition of a South Australian grassland. Austral Ecology 28:23-32.

Liancourt, P., K. Tielborger, S. Bangerter, and R. Prasse. 2009. Components of 'competitive ability' in the LHS model: implication on coexistence for twelve co-occurring mediterranean grasses. Basic and Applied Ecology 10:707-714.

Mayfield, M. M., and J. M. Levine. 2010. Opposing effects of competitive exclusion on the phylogenetic structure of communities. Ecology Letters 13:1085-1093.

McIntyre, S. 2008. The role of plant leaf attributes in linking land use to ecosystem function in temperate grassy vegetation. Agriculture, Ecosystems and Environment 128:251-258.

Messier, J., B. J. McGill, and M. J. Lechowicz. 2010. How do traits vary across ecological scales? A case for trait-based ecology. Ecology Letters 13:838-848.

Meziane, D., and B. Shipley. 1999. Interacting determinants of specific leaf area in 22 herbaceous species: effects of irradiance and nutrient availability. Plant Cell and Environment 22:447-459.

Ordonez, J. C., P. M. van Bodegom, J.-P. M. Witte, I. J. Wright, P. B. Reich, and R. Aerts. 2009. A global study of relationships between leaf traits, climate and soil measures of nutrient fertility. Global Ecology and Biogeography 18:137149.

Prober, S. M., K. R. Thiele, I. D. Lunt, and T. B. Koen. 2005. Restoring ecological function in temperate grassy woodlands: manipulating soil nutrients, exotic annuals and native perennial grasses through carbon supplements and spring burns. Journal of Applied Ecology 42:1073-1085.

R Development Core Team. 2012. R: a language and environment for statistical computing. R Foundation for Statistical Computing, Vienna, Austria. http://www. R-project.org/ 
Scougall, S. A., J. D. Majer, and R. J. Hobbs. 1993. Edge effects in grazed and ungrazed Western Australian wheatbelt remnants in relation to ecosystem reconstruction. Pages 163178 in D. A. Saunders, R. J. Hobbs, and P. R. Ehrlich, editors. Nature conservation 3: reconstruction of fragmented ecosystems. Surrey Beatty, Sydney, New South Wales, Australia.

Shipley, B., D. Vile, E. Garnier, I. J. Wright, and H. Poorter. 2005. Functional linkages between leaf traits and net photosynthetic rate: reconciling empirical and mechanistic models. Functional Ecology 19:602-615.

Sims, L., J. Pastor, T. Lee, and B. Dewey. 2012. Nitrogen, phosphorus and light effects on growth and allocation of biomass and nutrients in wild rice. Oecologia 170:65-76.

Standish, R. J., V. A. Cramer, and R. J. Hobbs. 2008. Land-use legacy and the persistence of invasive Avena barbata on abandoned farmland. Journal of Applied Ecology 45:15761583.

Stevens, J. C., D. J. Merritt, G. R. Flematti, E. L. Ghisalberti, and K. W. Dixon. 2007. Seed germination of agricultural weeds is promoted by the butenolide 3-methyl-2H-furo 2,3-c pyran-2-one under laboratory and field conditions. Plant and Soil 298:113-124.

Sultan, S. E., and F. A. Bazzaz. 1993. Phenotypic plasticity in Polygonum persicaria. 1: Diversity and uniformity in genotypic norms of reaction to light. Evolution 47:10091031.

Violle, C., B. J. Enquist, B. J. McGill, L. Jiang, C. H. Albert, C, Hulshof, V. Jung, and J. Messier. 2012. The return of the variance: intraspecific variability in community ecology. Trends in Ecology and Evolution 27:244-252.

Vitousek, P. M., H. A. Mooney, J. Lubchenco, and J. M. Melillo. 1997. Human domination of Earth's ecosystems. Science 277:494-499.

Westoby, M. 1998. A leaf-height-seed (LHS) plant ecology strategy scheme. Plant and Soil 199:213-227.

Wright, I. J., et al. 2004. The worldwide leaf economics spectrum. Nature 428:821-827.

Wright, I. J., and M. Westoby. 2000. Cross-species relationships between seedling relative growth rate, nitrogen productivity and root vs leaf function in 28 Australian woody species. Functional Ecology 14:97-107.

\section{Supplemental Material}

Appendix A

Supplementary field survey methods (Ecological Archives E095-035-A1).

Appendix B

Summaries of specific-leaf-area-environment models (Ecological Archives E095-035-A2).

Appendix C

Additional information for each of the modeled species (Ecological Archives E095-035-A3).

\section{Appendix D}

Minimum, mean, and maximum values of explanatory variables included in candidate models for each of the modeled species (Ecological Archives E095-035-A4).

\section{Appendix E}

A figure showing relationships between the magnitude of specific-leaf-area change from 2010 to 2011 and the change in relative and absolute abundance for each species over the same period, and photos showing the same location in 2010 (drier growing season) and 2011 (wetter growing season) (Ecological Archives E095-035-A5). 